Revue d'histoire de l'enfance « irrégulière »

Le Temps de l'histoire

Hors-série | 2007

Pages d'histoire, la protection judiciaire des mineurs, $\mathrm{XIX} \mathrm{X}^{\mathrm{e}} \mathrm{XX} \mathrm{X}^{\mathrm{e}}$ siècles

\title{
La patrouille des renards dans les cages à poules
}

L'Éducation surveillée et le scoutisme, 1937-1950

Jacques Bourquin

\section{(2) OpenEdition}

\section{Journals}

Édition électronique

URL : http://journals.openedition.org/rhei/3024

DOI : $10.4000 /$ rhei.3024

ISBN : 978-2-7535-1647-2

ISSN : $1777-540 \mathrm{X}$

Éditeur

Presses universitaires de Rennes

Édition imprimée

Date de publication : 1 juin 2007

Pagination : 283-294

ISSN : $1287-2431$

\section{Référence électronique}

Jacques Bourquin, "La patrouille des renards dans les cages à poules », Revue d'histoire de l'enfance " irrégulière » [En ligne], Hors-série | 2007, mis en ligne le 01 février 2010, consulté le 01 mai 2019. URL http://journals.openedition.org/rhei/3024 ; DOI : 10.4000/rhei.3024 


\section{La patrouille des renards dans}

\section{les cages à poules}

\section{L'Éducation surveillée et}

\section{le scoutisme, 1937-1950 ${ }^{(*)}$}

Pourquoi ce titre accrocheur et imagé ? Il correspond à une réalité institutionnelle, celle de l'Éducation surveillée de cette époque. Les activités scoutes que nous allons évoquer se déroulent dans quelques institutions pour mineurs relevant de l'Administration pénitentiaire jusqu'en 1945 et dont les quelques velléités de réforme ne remontent qu’à 1937. Les pupilles, on les appelle ainsi depuis 1927, dorment la nuit dans des "cages à poules ", sortes de cellules exiguës $(1,50 \mathrm{~m} \times 2 \mathrm{~m})$ et grillagées, dont le toit et la porte sont formés d'un treillis de fortes mailles, les autres parois sont en tôle épaisse. La cage à poules protège le pupille de ses voisins, mais permet aussi une surveillance de tous les instants. Lorsqu'en 1945 l'Éducation surveillée deviendra une direction indépendante de l'Administration pénitentiaire, il lui faudra encore de longues années pour se dégager de ces séquelles et les dernières cages à poules ne disparaîtront que vers la fin des années 1950.

Institutions longtemps contradictoires, les établissements d'éducation surveillée mêleront longtemps archaïsme pénitentiaire et innovation pédagogique. C'est dans ce second registre que l'on verra apparaître la référence au scoutisme dès 1937.

\section{Une première tentative de scoutisme à l'Éducation surveillée, 1937}

\section{Jacques Guérin-Desjardins}

Au lendemain de la guerre de 1914, cet étudiant passionné de psychologie, d'éducation et de scoutisme visite aux États-Unis une institution : la George Junior Republic ${ }^{(1)}$ où l'on s'occupe sur un mode très novateur d'enfants délinquants. Commissaire national des Éclaireurs de France de 1923 à 1936, il organise avec des éclaireurs unionistes des soirées récréatives au patronage Henri Rollet à Paris, qui reçoit des enfants placés par le tribunal pour enfants
(*) Article publié dans

Le scoutisme et la

rééducation dans l'immédiat après-guerre : lune de miel sans lendemain?, Documents de l'INJEP, $n^{\circ} 21,1995$.

(1) À partir d'un entretien de Michel Chauvière avec Jacques GuérinDesjardins, 1980. 
(2) Ibid. et adolescents de la Seine. C'est là qu'il rencontre le neuropsychiatre Georges Heuyer, dont le rôle sera important dans l'élaboration du secteur de l'enfance inadaptée. Parmi les éclaireurs qui interviennent avec Jacques GuérinDesjardins au patronage Henri Rollet, quelques adolescents dont les pères ont des positions importantes à la Justice : le fils du directeur de la prison de Fresnes, le jeune Baffos dont le père est président du tribunal d'enfants et d'adolescents de la Seine et le jeune Rucart dont le père député va être le garde des Sceaux du gouvernement de Front populaire en 1936. À la même époque, Jacques Guérin-Desjardins collabore au Comité des œuvres sociales, auquel participe Mme Brunschwig qui va devenir en 1936 sous-secrétaire d'État à l'Éducation nationale. En 1936, on est en pleine campagne de presse contre les bagnes d'enfants, le ministre Marc Rucart souhaite réformer ces établissements et c'est en accord avec Mme Brunschwig qu'il sollicite Jacques Guérin-Desjardins pour participer à l'élaboration de cette réforme. ${ }^{(2)}$

Marc Rucart lui demande de diriger un projet de formation pour les vingt futurs moniteurs-éducateurs qui auront comme mission de débuter la réforme de la maison d'éducation surveillée de Saint-Maurice à Lamotte-Beuvron. Ces stagiaires cédés par l'Éducation nationale sont volontaires et quelquesuns ont pratiqué le scoutisme, mais pour la grande majorité d'entre eux, il s'agit d'instituteurs surnuméraires sans expérience pédagogique. Apparemment, l'Éducation nationale, malgré le soutien de Mme Brunschwig, n'a collaboré que timidement avec la Justice.

La session a lieu à la prison de Fresnes en novembre et décembre 1936, elle dure cinq semaines. Y interviennent le psychiatre Georges Heuyer, le président du tribunal pour enfants et adolescents de Paris, mais aussi de nombreux scouts, dont André Lefevre - «Vieux Castor » qui va fonder quelques mois plus tard les CEMEA -, Pierre François, Georges Bertier, président des Éclaireurs de France et directeur de l'école des Roches, un inspecteur général de l'enseignement technique, J. Roumajon, qui sera un des théoriciens de la réforme de Saint-Maurice.

Cette formation est ponctuée par une visite de quelques jours au centre d'observation de Moll en Belgique fondé par l'instituteur Rouvroy au début du siècle, et qui apparaît comme un établissement modèle en matière d'enfance délinquante. (Il est à remarquer que dans ces tentatives de réforme de 
1936, on ne s’intéresse qu'aux établissements de garçons. Personne ne semble évoquer le sort des écoles de préservation de Clermont-de-l'Oise, de Doullens et de Cadillac, où l'Administration pénitentiaire reçoit les mineures.)

Début janvier 1937, les moniteurs-éducateurs sont envoyés à Saint-Maurice. Contrairement à son attente, Jacques Guérin-Desjardins n'est pas convié à les accompagner. $^{(3)}$

Introduire les références du scoutisme dans une institution pénitentiaire vieille de près de 70 ans, qui ne connaît que le dressage et la correction, était un pari impossible, d'autant plus que la direction et les personnels de SaintMaurice n’avaient pas été associés à une réforme uniquement impulsée par le ministre. Les moniteurs-éducateurs furent mal accueillis et le projet se révéla vite un échec. Ce fut rapidement l'évasion des jeunes et la démission des moniteurs-éducateurs. Le directeur de Saint-Maurice avait fait dire à ses personnels : "Ne vous inquiétez pas, les ministres changent, les directeurs restent... » La prédiction se révéla en partie fausse, car un an après, en 1938, un nouveau garde des Sceaux, Paul Reynaud, imposera une nouvelle tentative de réforme, il nommera un nouveau directeur, mais cette fois-ci, ce ne sera pas le scoutisme que l'on choisira comme référence, mais la formation professionnelle, l'apprentissage, sous l'influence de l'inspecteur général Roumajon auquel l'Administration pénitentiaire avait demandé une inspection de Saint-Maurice en 1935 et 1937 . L'heure du scoutisme n'avait pas encore sonné à l'Éducation surveillée!

\section{L'influence du scoutisme à l'Éducation surveillée, 1942-1950 \\ Les établissements de garçons : l'expérience de Saint-Hilaire}

Pendant la guerre, alors que l'Éducation surveillée dépend toujours de l'Administration pénitentiaire, deux établissements sont en cours de réforme : Saint-Maurice et Saint-Hilaire près de Saumur, et un troisième vient d'ouvrir en 1943 à Saint-Jodard près de Roanne.

Si Saint-Maurice est resté très fixé sur l'enseignement technique et ne s'ouvre pas au scoutisme, ce ne sera pas le cas des deux autres.

Un élément conjoncturel va favoriser, en particulier à Saint-Hilaire et Saint-Jodard, la rencontre avec le scoutisme, c'est le recrutement, à partir de fin 1942, de moniteurs-éducateurs qui entrent dans les services dépendant de
(3) Henri Gaillac, Les maisons de correction, Éd. Cujas, Paris, 1991 (rééd.), p. 308-309. 
(4) Les établissements

d'éducation surveillée

de l'époque sont soumis au système progressif, les pupilles débutent leur séjour à la section d'épreuve à caractère disciplinaire, puis, suivant l'amélioration de leur comportement, ils passent à la section normale puis d'honneur dont les régimes sont plus souples.

(5) « Le scoutisme à l'IPES de Saint-Hilaire ", in Pour l'enfance "coupable", octobredécembre 1945.
l'Administration pénitentiaire pour échapper au travail obligatoire en Allemagne : le STO. Ces nouveaux venus, très jeunes pour la plupart, viennent de secteurs très divers, ce sont des étudiants, des instituteurs, des chômeurs, des séminaristes... Bon nombre d'entre eux ont fréquenté le scoutisme et ont connu les chantiers de jeunesse. C'est à partir de ces deux modèles, qui ne sont pas sans points communs (l'amour de la nature, la vie en équipe, le respect du chef, le travail, les loisirs), qu'ils vont "inventer ", avec les jeunes pupilles de l'Éducation surveillée, les premiers rudiments d'une pédagogie qui inclut la vie en équipe, la loi, les loisirs.

Il est à remarquer qu'à la même époque dans le secteur associatif de la rééducation, qui est en train de se constituer, les premiers centres d'accueil sont ouverts souvent par d'anciens scouts. À Saint-Hilaire, le directeur François Dhallenne, le réformateur de 1938, voit dans le scoutisme un moyen de transformer l'esprit encore fortement pénitentiaire de son établissement. Un de ses adjoints, Victor Ventré, écrira en 1945, dans le numéro 253 du Chef, ce que fut le scoutisme à Saint-Hilaire pendant la guerre, " un scoutisme sur mesure destiné aux pupilles les plus méritants, ceux de la section d'honneur ». ${ }^{(4)}$

"Les garçons portent un uniforme scout car avec la tenue, ils changeaient d'âme. "

"Ils étaient aspirants deux à trois mois et devenaient éclaireurs le jour de leur promesse, ils recevaient à cette occasion un insigne, "obstacle à franchir", que nous avions trouvé dans la collection "signe de piste". "

"Ils choisissaient un patron, c'était souvent un grand explorateur, un aviateur... " ${ }^{(5)}$

Sur les deux cent quatre-vingts jeunes de Saint-Hilaire, il y avait vers 1943 jusqu'à quatre patrouilles et un clan de soutien de 18 à 21 ans, soit un total d'environ cinquante-huit jeunes. Le clan était dirigé par un jeune interne en médecine qui intervenait dans l'établissement et qui, après la guerre, aidera à faire le lien avec le scoutisme officiel.

Dans les archives de Saint-Hilaire, nous avons trouvé des comptes rendus de camps. En 1942, deux patrouilles, dont celle des Renards, ils sont dixhuit, quittent Saint-Hilaire pour un camp de quinze jours dans une propriété privée près de Thouars. Jusqu'alors, et cela depuis 1864, date d'ouverture de Saint-Hilaire, jamais aucune activité des colons puis des pupilles ne s'était 
déroulée hors des limites de la colonie ! Le scoutisme apparaissait comme une première brèche dans cet univers désespérément clos.

Victor Ventré nous apprend qu'au lendemain de la guerre, alors que l'Éducation surveillée a conquis son indépendance par rapport à l'Administration pénitentiaire, le scoutisme va être utilisé dans une fonction de postcure à la sortie de l'institution. "Les chefs à qui nous adressons nos garçons reçoivent une notice de renseignements, confidentielle et complète, sur lui afin de mieux le connaître, et de mieux l'aider à rester fidèle à sa promesse. "

Il semblerait que cette pratique fut éphémère, était-elle compatible avec la nouvelle ordonnance du 2 février 1945 sur l'enfance délinquante? Il semble que ce fut plus un vœu qu'une réalité, il est toutefois à remarquer que de nombreux chefs scouts furent au lendemain de la guerre délégués bénévoles à la liberté surveillée où ils eurent vraisemblablement un rôle de postcure.

Cette lettre qu'un jeune pupille de Saint-Hilaire adresse en février 1945 à son ancien éducateur ${ }^{(6)}$ peut apparaître comme une illustration de ce projet de " postcure » (plusieurs lettres de ce type ont été retrouvées).

"Cher ami,

"Vous avez dû trouver drôle que je vous écrivais plus. Ici en ce moment, j'ai quelques petits ennuis, j'ai cassé ma conduite, pour des raisons que je n'ose pas vous dire, j'ai passé en cour d'honneur et je suis descendu du clan. Mais je n'ai pas renoncé au scoutisme, dans un mois je passe en place à Saumur et là je pourrai m'enrôler dans une troupe. Vous ne m'écrivez plus, vous croyez peut-être que je vous ai oublié, loin de là des amis comme vous, on ne les oublie pas. Je vous ai fait une promesse, je la tiendrai. Un jour qui maintenant n'est pas loin, Claude D. et moi nous nous trouverons dans la même troupe à Paris. Je termine en vous serrant une poignée de main en scout.

Votre ami. " ${ }^{(7)}$

Le scoutisme à Saint-Hilaire et à Saint-Jodard semble ne pas avoir duré audelà de 1950, en tout cas nous n'en avons pas retrouvé de traces, et il n'existe pas dans les autres établissements, Belle-Île et Aniane, dont la réforme ne débuta qu'après 1945 .
(6) Cet éducateur recruté pendant la guerre pour lui permettre d'échapper au STO a regagné en novembre 1944 la « vie civile».

(7) Archives personnelles de Jean Cavozzi (ancien éducateur à Saint-Hilaire). 
(8) Béatrice Koppel, Marguerite B., une jeune fille en maison de correction, Paris, Hachette, 1987, p. 142.

(9) Jacques Bourquin, Béatrice Koeppel, "Deux contributions à la connaissance des origines de l'Éducation surveillée ", Cahiers $d u$ $C R I V, \mathrm{n}^{\circ} 2$, Vaucresson, octobre 1986, p. 81.

\section{Les établissements de filles : Cadillac et Brécourt}

La volonté de réformer des établissements de filles n’apparaît que tardivement à l'Éducation surveillée. En 1944, on parle toujours de maisons de préservation et les mineures croupissent dans ces institutions où la dureté du quotidien décrite par Béatrice Kœppel ${ }^{(8)}$ dans son ouvrage sur Cadillac n’a apparemment pas d'égal chez les garçons. Paul Lutz, magistrat au service de l'Éducation surveillée et ancien scout, évoque dès 1941 la nécessité de réformer ces institutions. C'est lui qui fera appel, fin 1943, pour réformer la maison de préservation de Cadillac, à Dominique Riehl, diplômée de psychologie, assistante du professeur Lagache à l'université de Clermont-Ferrand et ancienne cheftaine éclaireuse. Dominique Riehl, investie de cette nouvelle fonction en 1944, reçoit une « carte blanche " pour recruter ses collaborateurs. Elle va s'entourer d'une dizaine de jeunes femmes dont certaines sont issues du scoutisme, à l'exemple de son adjointe Geneviève Boutault, ancienne cheftaine, qui a dirigé une école de service social. ${ }^{(9)}$

Le projet qui s'élabore à Cadillac en 1944 avec des mineures dont beaucoup, récidivistes ou renvoyées des Bons Pasteurs, sont là depuis des années, est de créer un autre climat de vie, d'établir un autre type d'échange entre les adolescents et les personnels et, comme l'exprime Paul Lutz, " de ne plus considérer les pensionnaires comme des perverses constitutionnelles, des inamendables ». L'œuvre est difficile ! L'idée est tout d'abord de constituer avec les jeunes des équipes de vie où les éducatrices, recrutées par Dominique Richl, remplaceront progressivement les personnels pénitentiaires.

Geneviève Boutault évoque « chaque équipe de huit filles confiées à une éducatrice, l'équipe dispose d'un local où se déroulent les activités scolaires, les loisirs dirigés, les veillées, on y prend même ses repas ". Linstitution abandonne partiellement les grands réfectoires, les salles de classe. Il y a, précise Geneviève Boutault, "quelque chose qui s'apparente à la fois au scoutisme et à la famille ". "Ainsi, à Cadillac, la veillée est beaucoup plus une veillée familiale où chacune s'occupe selon son gré : couture, tricot, lecture, jeux, qu'une veillée scoute où chacune est intégrée dans l'activité commune..., nos filles ont besoin de savoir d'abord ce qu'est une atmosphère paisible, alors que les souvenirs familiaux sont presque toujours pour elles : coups, disputes, séparation. " Mais après la veillée où l'on chante, les mineures sont enfermées dans leurs cellules. 
Au tout début de l'expérience, Dominique Rhiel et Geneviève Boutault avaient pensé faire appel à une cheftaine de l'extérieur pour « séparer le scoutisme de l'internat ». Dans ce contexte encore semi-pénitentiaire, c'était difficile, il s'agissait donc d'un scoutisme très centré sur l'institution avec néanmoins quelques ouvertures : un premier camp dans les Landes vers 1945-1946.

La réforme de Cadillac s'avérait difficile. Béatrice Koppel évoque toutefois " la rigueur pénitentiaire qui se dénoue par un compromis apparemment harmonieux avec le formalisme scout ". L'institution fermera définitivement en 1951 et, dès 1947, Dominique Riehl et Geneviève Boutault ouvraient à l'Éducation surveillée un établissement de rééducation pour filles : l'IPES de Brécourt, à une dizaine de kilomètres de Pontoise. On peut dire de Brécourt qu'il fut, pour Dominique Riehl et Geneviève Boutault, un laboratoire pour la mise en place de la rééducation des filles délinquantes, le scoutisme y est évoqué jusqu'en 1964, mais avec beaucoup de réserves.

En 1946, dans une des conférences du Méridien, organisée par le foyer universitaire du scoutisme français sur le thème de l'enfance délinquante (les conférences ont lieu à la Sorbonne), Jean Jousselin, ${ }^{(10)}$ secrétaire général du scoutisme français, parle des liens possibles entre le scoutisme et la rééducation :

«Le scoutisme doit jouer son plein rôle dans les milieux délinquants..., il est une société fraternelle où l'on donne au jeune l'occasion de recommencer sa vie, de reconstruire un monde nouveau, dans la mesure où il reconstruit ce monde, on lui permet de reconstruire sa propre personne... Tout le passé n'existe plus, ni pour la patrouille ni pour les chefs... il est dans une société nouvelle..., il dénoncera tous ses complexes d'infériorité, tous ses troubles d'opposition. " Dans les traits qui dominent le scoutisme, Jean Jousselin privilégie la notion d'honneur : "Cela n'a rien à voir avec le sentiment moral, le gangster peut être un homme d'honneur, ne pas vendre son copain... L'honneur, c'est quand on peut avoir confiance en moi, c'est le sens de la promesse scoute..., l'enfant devient responsable, il n'a plus le droit d'être égoïste, il est obligé de donner à ses camarades ce qu'il refuse à la société. "Enfin, conclut Jean Jousselin : "Le scoutisme est une méthode active..., la confiance stimule l'enfant à agir... il est l'apprentissage de la vie sociale... un merveilleux moyen de salut pour les jeunes désadaptés. »
(10) Jean Jousselin, Le problème de l'enfance délinquante, Paris, ESF, 1946, p. 145-151. 
(11) Fonds Brisset,

versement $n^{\circ} 20000111$, Centre des archives contemporaines, Fontainebleau.
À cette image, peut-être un peu idyllique, du scoutisme, l'institution de Brécourt se situe sur un plan beaucoup plus pragmatique en introduisant de fortes réserves, liées à la personnalité de la jeune délinquante et aux contraintes de la rééducation.

Le projet pédagogique de Brécourt, ${ }^{(1)}$ et en cela il s’inscrit bien dans les priorités de l'Éducation surveillée de l'après-guerre, repose sur des points forts :

- «l'éducation par l'exemple";

- « une rééducation individuelle, à base de formation du caractère et d'apprentissage à la discipline sociale »;

- « l'apprentissage de la vie familiale », ce qui est plus spécifiquement féminin dans le projet de l'Éducation surveillée ;

- « une formation professionnelle très poussée (industrielle, agricole, horticole) »;

- « une éducation du sens moral et de la responsabilité ».

À l'exception de l'apprentissage de la vie familiale et de la formation professionnelle, on y retrouve des principes du scoutisme. Toutefois, ce projet se termine par :

- «créer de bonnes habitudes, voire des automatismes ", principe qui nous éloigne des pédagogies actives dont le scoutisme est porteur ;

- « apprendre aux élèves à rembourser à la société des dépenses consenties pour elles".

On peut être surpris par cette formule, les filles sont coupables, elles doivent " payer ", alors que, depuis les années 1930, le mythe de l'enfance coupable fait place à celui de l'enfance victime, malheureuse.

Dans ce même projet, Dominique Riehl, la directrice de Brécourt, s'attarde sur la personnalité des mineures délinquantes de l'établissement.

« Pour $95 \%$ d'entre elles, il faut souligner la tendance à la paresse, à la vie facile, le penchant à la débauche, soit comme goût, soit comme moyen, l'angoisse, le désordre, le laisser-aller, la faiblesse de la personnalité. »

Un regard bien différent de celui de Jean Jousselin sur les jeunes délinquants, une violence de traits que l'on ne retrouve pas dans les projets d'établissements de garçons de l'Éducation surveillée de l'époque. La marginalité de l'adolescente apparaît bien plus intolérable que celle du garçon. On conçoit alors combien le projet du scoutisme ne sera utilisé qu'avec beaucoup 
de réserves à Brécourt. Dans une conférence qu'elle fait en juin 1949 à de jeunes cheftaines, au centre d'observation de Chevilly-Larue, sur le thème " ce qu'apporte le scoutisme féminin à la rééducation ", Geneviève Boutault évoque ces réserves. Trois critères lui paraissent nécessaires pour utiliser le scoutisme avec une adolescente : «le volontariat », «le mérite », « le profit que la jeune peut en tirer ", mais « tous les enfants ne sont pas faits pour le scoutisme... la méthode doit avec nos filles être adaptée avec beaucoup de prudence ».

À Cadillac, la confiance que l'on a dans la jeune est très limitée : «Elle est difficile, car si le sens de l'honneur reste vivant chez les filles délinquantes, il consiste surtout à se soutenir mutuellement en toutes circonstances, c'est la loi du milieu, pour la plupart d'entre elles le grand manquement à l'honneur serait de faire échouer une évasion..., il devient alors difficile de se fier à leur parole. »

Voilà qui nous éloigne des propos de Jean Jousselin :

"Le principe d'auto-éducation, ajoute Geneviève Boutault, est difficile à expliquer avec des délinquantes qui sont en général en révolte contre l'organisation sociale, sans distinguer le bien du mal... prendre en main leur propre rééducation n'est possible qu'après une longue évaluation. "

Geneviève Boutault reconnaît que cette responsabilisation des jeunes est formatrice, mais « il ne faut pas que le poids des responsabilités écrase les filles». C'est une des raisons pour lesquelles on ne retient ni la loi scoute, ni la promesse scoute, contrairement aux établissements de garçons : Saint-Hilaire, SaintJodard. Il ne faut pas donner à l'adolescente une source d'échec supplémentaire, dans la mesure où elle n'est pas encore capable de tenir sa parole. Le scoutisme, avec son modèle fondé sur l'exemple et un certain élitisme moral, ne peut être pour l'adolescente de Cadillac ou de Brécourt qu'un aboutissement.

En fait, la réponse apportée par le scoutisme à Brécourt semble moins concerner les adolescentes que les éducatrices pour lesquelles, précise Geneviève Boutault, il est " une préformation, il leur apporte l'amour de l'enfant, le désir de travailler en équipe, la loyauté, la conscience professionnelle, la joie, la bonne humeur... toutes qualités qui aident à surmonter les difficultés du métier ".

Recruter des cheftaines, c'est sélectionner des personnes qui ont une pratique avec des jeunes mais qui représentent surtout une sorte d'élite morale, une image rassurante et disciplinée de l'adulte. S'il y a eu de nombreuses cheftaines scoutes 
(12) Lettre d'Henri

Michard à Jacques

Bourquin, 17 janvier 1994.

(13) Entretien de Jacques Bourquin avec Paul Lutz, 9 novembre 1993.

(14) Système pédagogique instauré à SaintMaurice en 1945 qui donne une certaine représentativité aux élèves de l'IPES dans le choix des loisirs et de la vie quotidienne. dans les débuts de Cadillac et Brécourt, on peut se demander s'il y a eu réellement une pratique scoute dans les projets de ces institutions.

À Brécourt comme dans d'autres institutions du même type, malgré un réel souci d'éducation, la référence pénitentiaire est toute proche, on est dans des projets qui relèvent encore fortement du redressement, de la normalisation avec un lointain projet de reclassement social, subsiste encore l'idée de dette à payer à la société, de faute..., le scoutisme ne pouvait qu'avoir du mal à s'y frayer un chemin, et sa réponse pouvait apparaître comme bien imparfaite.

Geneviève Boutault concluait son propos en disant : "Le scoutisme ne doit pas entrer en conflit avec la ligne éducative, la prépondérance doit rester à l'éducation. »

\section{La position officielle au lendemain de la guerre \\ Les nouveaux éducateurs}

On ne peut nier que le scoutisme ait été une référence chez les pionniers de la rééducation à l'Éducation surveillée. Toutefois, la nouvelle direction de l'Éducation surveillée ne l'inscrit pas dans ses projets de réforme. Henri Michard $^{(12)}$ et Paul Lutz, ${ }^{(13)}$ qui furent tous deux inspecteurs de l'Éducation surveillée en 1945 , nous l'ont confirmé.

Le règlement provisoire des IPES d'octobre 1945, en privilégiant la réinsertion sociale par la formation professionnelle, demeure dans une pédagogie de type progressif héritée de la Pénitentiaire. Le mérite, que l’on ne définit guère, permet au mineur de progresser dans l'institution en faisant un cheminement, de la section d'épreuve de type disciplinaire à la section d'honneur plus libérale. Les expériences inspirées du scoutisme relèvent surtout d'initiatives personnelles, en une époque où chacun apportait ce qu'il savait faire.

Le scoutisme à Saint-Hilaire, la "république » ${ }^{(14)}$ à Saint-Maurice permettront d'assouplir le système progressif beaucoup plus fondé sur l'adaptation à l'institution que sur l'évolution personnelle du jeune.

En 1946, l'inspecteur Pinatel, évoquant les doctrines et conceptions nouvelles de l'Éducation surveillée, critique la référence abusive au scoutisme dans certains établissements d'éducation surveillée et dans ceux du secteur associatif, en particulier Kergoat. Il s'interroge sur les vertus et les limites du scoutisme dans le cadre de la rééducation des mineurs délinquants. Il y voit 
une résurgence des théories du XIXème siècle concernant les colonies agricoles et le retour à la terre. "Le scoutisme conviendra-t-il à assumer le redressement d'enfants d'origine urbaine transplantés à la campagne, suffira-t-il à changer la mentalité des enfants des villes pour leur faire aimer la vie des champs, non à la manière scoute, mais le labeur paisible du paysan...? " Et il ajoute : "Le scoutisme, malgré ses qualités d'aide au développement du sens des responsabilités, du sens de l'effort, de la mise en confiance de l'enfant... ne serait qu'un artifice générateur de bien des désillusions, mieux vaut que le jeune revienne à sa libération muni d'un CAP. " (15)

Henri Joubrel ${ }^{(16)}$ réagit à cet article en affirmant « que le scoutisme adaptait fort bien la notion de self government et celle de la progressivité ». Lorsque l'Éducation surveillée commencera, dès 1947 à Savigny, à Marly-le-Roi, puis en 1951 à Vaucresson, à mettre en place une formation d'éducateurs, Henri Michard préférera au scoutisme la référence aux méthodes d'éducation populaire " considérées comme un moyen de donner une dimension plus éducative aux séances d'activités dirigées ". La pédagogie des internats d'Éducation surveillée fut traversée, jusqu’à la fin des années 1950, par les techniques pédagogiques issues du mouvement Peuple et Culture dont les objectifs pouvaient apparaître plus liés aux intérêts et aux besoins d'émancipation de jeunes issus de la classe ouvrière. Parmi les deux cents monographies rédigées par les élèves éducateurs des promotions de Vaucresson entre 1951 et 1962, une seule se référait au scoutisme.

La réserve de l'Éducation surveillée à l'égard du scoutisme était peut-être liée à la difficulté qu'il y avait à l'adapter à la pédagogie des délinquants dans un contexte qui restait encore tributaire de l'esprit pénitentiaire. Un autre argument pour expliquer cette réserve nous semble aussi venir de l'origine institutionnelle et culturelle des premiers éducateurs de l'Éducation surveillée. En $1945,{ }^{(17)}$ deux cent trente-huit d'entre eux proviennent, après sélection, de l'Administration pénitentiaire où ils étaient employés depuis quelques années comme moniteurs ou moniteurs-éducateurs. La petite centaine recrutée entre fin 1942 et début 1944 parmi des étudiants, des chômeurs qui veulent échapper au STO, ne sera plus qu'une dizaine en 1945. Ce sont surtout eux qui ont été porteurs du modèle scout. Les recrues de 1945 dans le cadre du nouveau statut des personnels de l'Éducation surveillée, bacheliers pour les deux tiers
(15) Jacques Pinatel,

« La doctrine de l'Éducation surveillée devant une conception nouvelle ", Revue de science criminelle et droit pénal, 1946.

(16) Henri Joubrel, « Le problème de l'Éducation surveillée ", Revue de science criminelle et droit pénal, 1947.

(17) Ibid., p. 44. 
d'entre eux, cherchent du travail au lendemain de la guerre. Certains reviennent de camps de prisonniers, parmi eux des gens qui avaient souhaité être instituteurs et qui n'avaient pu, du fait de la guerre, entrer à l'École normale. Globalement, il s'agit d'un milieu de gens issus ou se destinant à la fonction publique, ils sont assez proches des classes populaires avec une assez forte référence à la laïcité, beaucoup d'entre eux n’ont pas rencontré le scoutisme, peut-être plus les patronages.

La question avait été : peut-on adapter le scoutisme à la rééducation des délinquants ? L'Éducation surveillée répondit plutôt négativement pour des raisons d'ordre institutionnel, culturel, idéologique et professionnel. 